\section{Importancia de la interacción social en la retroalimentación, según residentes de Psiquiatría}

\author{
PATRICIA RUBÍ G. ${ }^{1, a}$, LUIS DE LA BARRA V. ${ }^{2, \mathrm{~b}}$, HARDY SCHAEFER A. 2,b,c
}

\section{The opinion of psychiatry residents about social interaction during the feedback in medical education}

Background: In Medical Education, feedback is an instrument that informs the trainees about their learning process and how to make the necessary changes. Feedback enables learners to monitor their progress and provides direction for improvement. Effective feedback is useful to improve the performance, clinical skills, communication skills, and treatment of patients. It is essential when teaching clinical interview skills to psychiatry residents. Feedback necessarily involves social interaction between teachers and trainees. Aim: To analyze the perception of psychiatry residents about social interaction during the feedback received during their training. Material and Methods: Qualitative study, using the Grounded theory method. Nine interviews were performed to adult psychiatry residents of the University of Concepcion and one to a recently graduated psychiatrist. Data were analyzed using open coding. Results: We found four emerging categories: Socialization, roles and hierarchy, invitation to integration and teacher involvement. Conclusions: The effectiveness of feedback lies in the establishment of a minimal teacher-resident social relationship, in which feedback occurs naturally.

(Rev Med Chile 2016; 144: 751-756)

Key words: Education, graduate; Education, Medical; formative Feedback; Interpersonal Relations.

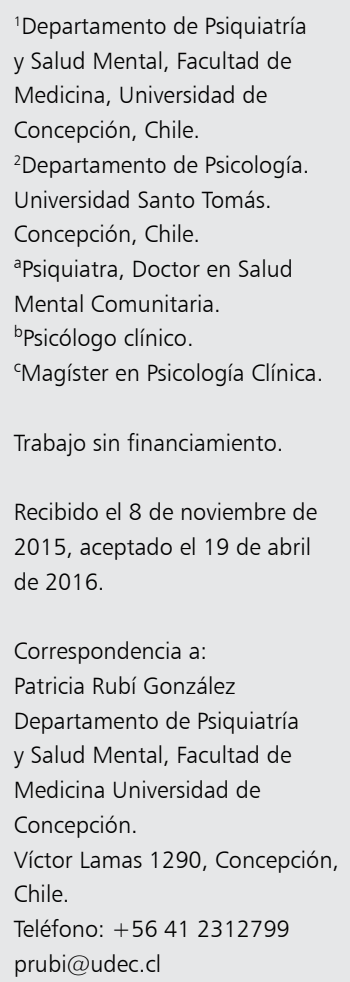

\section{I}

a retroalimentación en Educación Médica es una herramienta fundamental para la formación de hábitos y actitudes en los estudiantes. Se refiere al proceso de informar al alumno sobre su desempeño en diversos aspectos (cognoscitivos, actitudinales y en la evaluación de sus destrezas técnicas) para mejorar su rendimiento futuro, enfatizando aquellos aspectos positivos y señalando vías para enmendar sus falencias ${ }^{1}$.

La evidencia empírica ha demostrado que la retroalimentación constructiva y eficaz mejora los resultados del aprendizaje ${ }^{2}$, incluyendo mejores notas en la evaluación en estudiantes universitarios, mejoras en el rendimiento clínico y competencias clínicas (por lo menos a corto plazo) en médicos que están realizando una pasantía $a^{3,4}$, y avances en las habilidades de comunicación y trato con los pacientes en estudiantes de Medicina ${ }^{5,6}$.

Tal como sucede en pregrado, la formación de especialistas ocurre en su mayoría en situaciones clínicas, por lo que supervisores y residentes reconocen que la retroalimentación es un componente crucial para el aprendizaje en estos contextos ${ }^{7}$. Los residentes están en proceso de desarrollar las capacidades de autoevaluación y autopercepción de sus habilidades, lo que les servirá para el resto de su vida profesional y allí es donde la retroalimentación juega un rol fundamental. Sin embargo, estudios muestran que un alto porcentaje de residentes refiere no haberla recibido nunca o 
de manera infrecuente ${ }^{8}$. Otros mencionan que, lamentablemente, los docentes no dedican el tiempo suficiente para observar su desempeño ni para entregar una retroalimentación útil ${ }^{9}$.

En la respuesta del residente a la retroalimentación se describe que influye principalmente la credibilidad y el rol apoyador del docente ${ }^{10}$. Por otro lado, la falta de confianza o respeto por el docente, la percepción de falta de experiencia del docente, la falta de observación directa del desempeño, las pocas habilidades de comunicación del docente, y la actitud incómoda del docente al entregar la retroalimentación, son factores que hacen que se considere la retroalimentación inválida ${ }^{11}$.

Dentro de los factores que se han asociado a una mejor percepción de la efectividad de la retroalimentación por parte de alumnos de medicina y residentes, se incluyen también: la especificidad de la información, las sugerencias de un plan de acción para mejorar el desempeño, el momento oportuno de recibirla tras el desempeño, una presentación sin prejuicios y la oportunidad dada para responder ${ }^{10}$. Lamentablemente, existe escasa información en la literatura respecto a la importancia que tiene la interacción social entre residente y docente durante la retroalimentación y cómo ésta influye en la motivación y finalmente en el aprendizaje durante la especialización.

Este estudio tiene por fin determinar las percepciones y la subjetividad implicada, de parte de los residentes, sobre el proceso de retroalimentación recibida durante la formación de especialistas en Psiquiatría del adulto en la Universidad de Concepción, determinando atributos y características que inciden en la retroalimentación.

\section{Metodología}

Este estudio se adscribió a un paradigma de investigación constructivista-construccionista, a una metodología hermenéutica-dialéctica, cuyo diseño fue cualitativo y utilizó la Grounded Theory ${ }^{12,13}$ como tipo de investigación. Esta metodología resulta especialmente apropiada para acceder a los procesos subjetivos desde la visión de los propios actores involucrados.

$\mathrm{Al}$ aplicar los procedimientos de la Grounded Theory, se realiza una labor inductiva, disponiendo de un diseño metodológico flexible, que permite integrar la información inesperada y contrastar sucesivas hipótesis ${ }^{14}$. Se trabajó con categorías emergentes, y con las categorías desarrolladas a partir de los datos se pudo realizar un análisis descriptivo o relacional. El producto final de este proceso inductivo consiste en un conjunto de conceptos relacionados entre sí, que permite dar cuenta de las cualidades del objeto de estudio. Se continuó de este modo hasta lograr la saturación teórica ${ }^{15}$.

Se realizó un muestreo intencionado, de carácter no probabilístico, dado que la elección de los participantes se determinó a partir del objetivo del estudio. La población escogida para el estudio fueron los residentes de $2^{\circ}$ y $3^{\circ}$ año de la especialidad de Psiquiatría del adulto de la Universidad de Concepción, y egresados de la especialidad de los últimos dos años (2012-2013).

La recolección de información se realizó mediante entrevistas en profundidad de naturaleza narrativa-episódica, donde la naturaleza narrativa de la entrevista avanza de la descripción a la argumentación explicativa de fenómenos ${ }^{16}$. La entrevista se centra en los episodios relativos a experiencias que son relevantes al fenómeno de estudio, oscilando entre narraciones orientadas a episodios y argumentaciones más cercanas a un conocimiento conceptual.

Se realizaron diez entrevistas en profundidad, a nueve residentes y un egresado del último año, de los cuales seis fueron hombres y cuatro fueron mujeres. Todos los participantes fueron voluntarios y firmaron previamente un consentimiento informado.

La información textual recopilada se manejó de manera confidencial y fue analizada mediante el método de comparación constante, el que no hizo necesario la continuación del muestreo, ya que se alcanzó la saturación teórica de las categorías conceptuales.

\section{Resultados}

A partir de la codificación abierta se obtuvieron 4 categorías, con sus respectivas subcategorías, las que se describen en la Tabla 1 y son ilustradas mediante viñetas (citas textuales). Cada viñeta es acompañada de un número que identifica el informante $(\mathrm{P})$ y otro número que indica el párrafo de la entrevista donde se ubicaba (PF).

Estas categorías de retroalimentación y relaciones sociales agruparon aquellos significados que evidenciaron la relación existente entre la 
Tabla 1. Retroalimentación y relaciones interpersonales

\begin{tabular}{|c|c|c|}
\hline Categorías & Subcategorías & Viñetas ilustrativas \\
\hline \multirow[t]{5}{*}{ Socialización } & $\begin{array}{l}\text { Importancia de la } \\
\text { relación social }\end{array}$ & $\begin{array}{l}\text { "yo creo que tener coloquialmente 'buena onda' es como tener esa } \\
\text { confianza, uno generalmente la va obteniendo fuera del ámbito acadé- } \\
\text { mico, uno esa confianza la va obteniendo porque uno conversa fuera } \\
\text { del hospital o dentro del mismo hospital, pero fuera de una instancia } \\
\text { docente" (P: 5, PF: 57-58) }\end{array}$ \\
\hline & $\begin{array}{l}\text { Frecuencia de } \\
\text { interacciones }\end{array}$ & $\begin{array}{l}\text { "y claro eso se va conformando en los distintos encuentros que uno tiene } \\
\text { en el pasillo, 'hola cómo estás' E: ¿Se va como rompiendo el hielo y eso } \\
\text { es bueno para el feedback después? P: si" (P: 5, PF: 64) }\end{array}$ \\
\hline & $\begin{array}{l}\text { Sensación de confianza } \\
\text { con los docentes }\end{array}$ & $\begin{array}{l}\text { "entonces eso también depende de la confianza que tenga con el docen- } \\
\text { te, yo creo que es súper importante para el tema del feedback porque } \\
\text { si no hay confianza yo tampoco voy a poder entrevistar libremente" (P: } \\
\text { 5, PF: 56-57) }\end{array}$ \\
\hline & $\begin{array}{l}\text { Credibilidad e influencia } \\
\text { de los mensajes }\end{array}$ & $\begin{array}{l}\text { "por ejemplo yo tengo más confianza con unos docentes más que con } \\
\text { otros, y si alguno de estos docentes que no tengo confianza me dicen } \\
\text { 'sabes, estás haciendo esto cuestión mal' para mí va a ser una reacción } \\
\text { diferente a que me diga uno más cercano que yo pensaré 'sabes, capaz } \\
\text { que tenga razón'" (P: 3, PF: 43-44) }\end{array}$ \\
\hline & Interacción negativa & $\begin{array}{l}\text { "que uno siempre está ahí en tono como severo, como imponer sus } \\
\text { ideas, sus puntos de vista siendo que la psiquiatría es súper amplia, hay } \\
\text { distintos puntos de vista, distintas corrientes, los diagnósticos general- } \\
\text { mente varían de un psiquiatra a otro y a veces esas discordancias con } \\
\text { algunos docentes uno las puede conversar, y con otros uno sabe que } \\
\text { puede ser complicado meterse" (P: } 5 \text {, PF: } 42 \text { ) }\end{array}$ \\
\hline \multirow[t]{4}{*}{ Desjerarquización } & Horizontalidad & $\begin{array}{l}\text { "claro, exactamente, es como estar en un mismo plano, eso también } \\
\text { genera confianza y eso hace que uno esté más abierto a cualquier tipo de } \\
\text { sugerencia o crítica que el docente le pueda hacer a uno" (P: 5, PF: 41-42) }\end{array}$ \\
\hline & Vulnerabilidad & $\begin{array}{l}\text { "creo que la forma no era la más adecuada, creo que la retroalimentación } \\
\text { se debe hacer personal, no a través de grupos y en el fondo no sé si la } \\
\text { palabra es 'humillar', en el fondo es exponer a la persona" (P: 7, PF: 5-6) }\end{array}$ \\
\hline & Miedo a la expresión & $\begin{array}{l}\text { "al principio cuando a ti te preguntan o cuando uno viene llegando a la } \\
\text { beca, uno siempre calla qué te paso a ti con el paciente, uno calladito dice } \\
\text { así como 'nada', así como 'no la voy a embarrar', con temor" (P: 4, PF: 28) }\end{array}$ \\
\hline & $\begin{array}{l}\text { Desbalance de poder en } \\
\text { la situación interpersonal }\end{array}$ & $\begin{array}{l}\text { "tiene que adscribirse al pensamiento del docente casi al pie de la letra y } \\
\text { las pocas veces que uno intenta argumentar desde lo que sabe o desde } \\
\text { la experiencia de uno, la respuesta que tiene es que 'él es el profesor" } \\
\text { (P: 9, PF: 10-11) }\end{array}$ \\
\hline $\begin{array}{l}\text { Invitación a la } \\
\text { integración }\end{array}$ & $\begin{array}{l}\text { Expresión de interés de } \\
\text { inclusión }\end{array}$ & $\begin{array}{l}\text { "en general me pasa cuando conozco al docente, cuando logro más ad- } \\
\text { miración por el docente y percibir que al docente le importa mi cambio, } \\
\text { yo como que me esfuerzo en lograr el cambio" (P: 8, PF: 60-61) }\end{array}$ \\
\hline $\begin{array}{l}\text { Involucración del } \\
\text { docente }\end{array}$ & Acompañamiento & $\begin{array}{l}\text { "él se daba el tiempo de revisarlo y cada cierto tiempo nos llamaba y nos } \\
\text { decía cuáles eran nuestras falencias y las dejaba escritas y después nos } \\
\text { daba instancias de volver a escribirlas y volver a mostrárselas, yo creo que } \\
\text { eso es súper, súper bueno" (P: 10, PF: 24) }\end{array}$ \\
\hline
\end{tabular}

retroalimentación y el ámbito de fenómenos de las relaciones humanas.

\section{Socialización}

Corresponde a una especificidad de contenidos que reflejaron cómo la interrelación que se da entre residentes y docentes ocurre como un paulatino socializar, en el cual, a razón de los sucesivos encuentros, va desarrollándose una mayor familiaridad que hace sostenible las interacciones que deben desarrollarse de ordinario entre estos actores. 
Los residentes valoraron la frecuencia de interacciones, la que influye en la sensación de confianza con los docentes, entendido como un conocimiento mínimo del otro, el que es reconocido por una mayor fluidez de las interacciones, tranquilidad para hablar libremente, así como también un mayor potencial de influencia de los mensajes recibidos. Es presentado como un fenómeno que parece sustentar las interacciones y coordinaciones que deben tener lugar entre residentes y docentes. Esta socialización incide sobre la credibilidad e influencia de los mensajes del docente, los que influyen más fácilmente en el desempeño y en la motivación al cambio del aprendiz.

Los residentes también mostraron preocupación respecto a la interacción negativa, a la anulación del otro en la relación o directamente a la percepción de rechazo de parte de los docentes, y cómo ésta afecta negativamente el proceso de retroalimentación, la motivación y el aprendizaje.

\section{Desjerarquización}

Algunas características de la relación social con el docente parecieron ser comprendidas por los residentes en el contexto de ciertas dinámicas sociales o por características culturales del contexto de estudio, específicamente los roles y jerarquías preestablecidas.

Los residentes destacaron significados asociados al respeto en la interacción docente-residente, como una característica deseada en la retroalimentación. Esta idea en las entrevistas finalmente decantó en la noción de horizontalidad, es decir, la relación tutor-aprendiz se define como carente de jerarquía, o dicho de otro modo, como conversaciones entre iguales.

Se señaló una vulnerabilidad asociada a la experiencia social de ser retroalimentado, dando a entender que el someterse a una situación de este tipo deja al residente expuesto frente al otro. De forma paralela, se evidenció un miedo a la expresión, es decir, un temor a expresar su opinión frente a situaciones de retroalimentación, asociado a la posibilidad de una eventual consecuencia negativa en la situación en curso, en términos de una sanción interpersonal, o al comprometer la actitud del docente en futuros encuentros o evaluaciones. En el mismo sentido, se señaló una situación de desbalance de poder en la situación interpersonal, que rige las relaciones académicas entre docentes y residentes, dinámica que afecta negativamente la situación de retroalimentación.

\section{Invitación a la integración}

Otra subcategoría que articula la idea de socialización es la que incluyó los significados de invitación e integración a una comunidad, en este caso, al grupo de psiquiatras. Los residentes percibieron la entrega de retroalimentación como una expresión de interés de parte del docente de incluirlo en su grupo de pertenencia. Esta invitación a la integración favorecería el proceso de retroalimentación y la motivación al aprendizaje.

\section{Involucración del docente}

De las citas se desprende que los residentes ponen atención sobre los comportamientos del docente que van delineando la relación que comparten, haciendo una deducción de la calidad de la vinculación entre ambos, a partir del interés percibido en el docente en su aprendizaje. Por otra parte, las situaciones concretas de retroalimentación fueron significadas como un punto en un proceso de elaboración de una relación entre docente-residente, apareciendo como relevante el seguimiento que hace el docente del progreso del aprendizaje del residente, a partir de las instancias de retroalimentación. Así, surgieron las ideas de acompañamiento, compromiso o disponibilidad del docente a través del tiempo.

\section{Resultados relacionales}

Una de las comprensiones más importantes a partir de los análisis descriptivos fue que, desde la experiencia de los participantes, la calidad o éxito de la retroalimentación recae en el establecimiento de una relación social mínima entre los actores, es decir, entre docente y residente. El desarrollo de la idea apuntó más bien a la proximidad social y al asentamiento progresivo de una interacción colaborativa, lo que no es necesariamente un vínculo afectivo o una amistad propiamente tal, sino un conocimiento social mínimo que valida el acercamiento necesario para participar del juicio de la imagen personal del otro.

Los resultados mostraron que una modalidad de interacción entre docente y residente, basada en la horizontalidad y en el involucramiento conjunto, permite que el retroalimentar resulte natural y fundamental para el éxito práctico de esta situación de aprendizaje. 


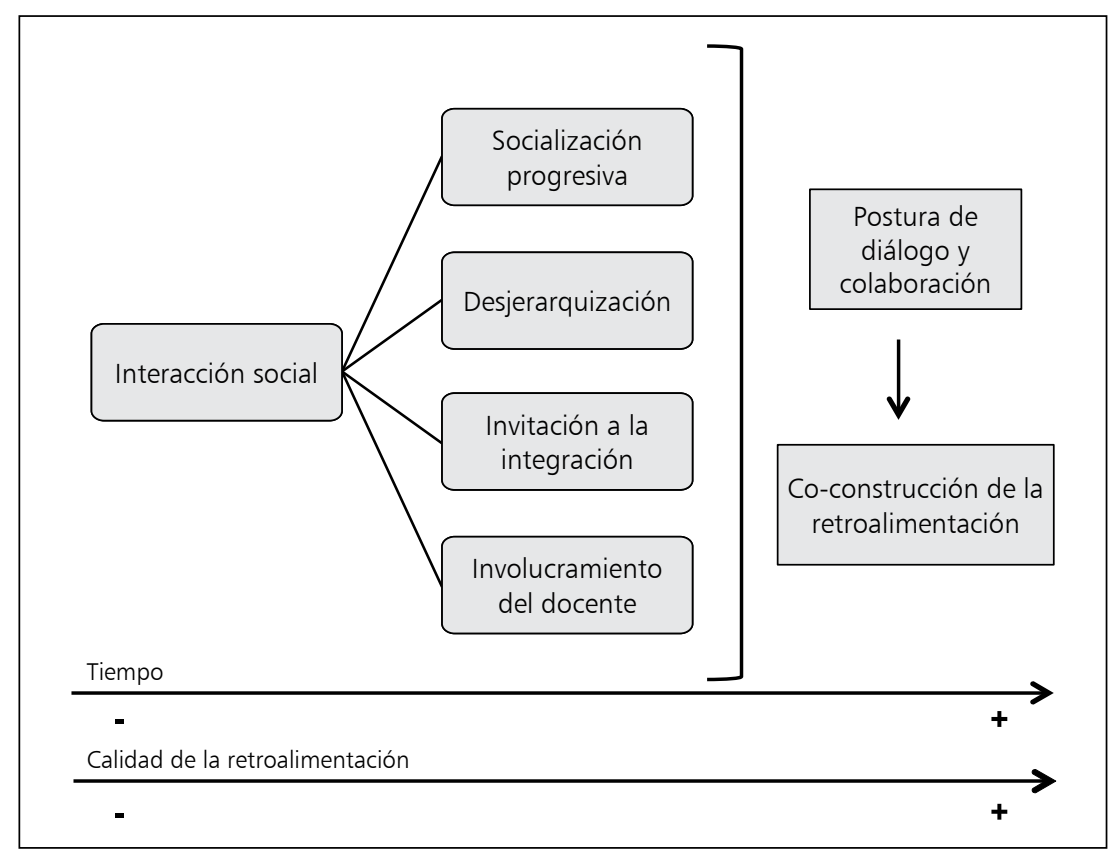

Figura 1. Interacción social colaborativa.
Esta configuración interpersonal se caracteriza porque el residente y el docente se sitúan de manera colaborativa para abordar algo, o se ocupan conjuntamente de una situación en particular. La posibilidad de discriminación en un ámbito práctico compartido (atención de pacientes) es lo que permite calificar al involucramiento como un escenario que favorece el aprendizaje, en donde el residente tiene la posibilidad de ser un participante activo de éste.

Las ideas relacionadas en este punto se pueden visualizar en la Figura 1.

\section{Discusión}

La retroalimentación, de acuerdo a la percepción subjetiva de los residentes de Psiquiatría de la Universidad de Concepción, requiere una plataforma de relaciones interpersonales entre el supervisor y los residentes en formación, la cual debe caracterizarse por una horizontalidad, una invitación a la integración y un involucramiento de parte del docente, o dicho de otro modo, formas de interacción que promuevan el diálogo y una actitud colaborativa entre los actores. En otras palabras, según el modo como las experiencias de retroalimentación son percibidas, los sujetos definen y enfatizan el papel central de la interacción social para una efectiva retroalimentación, es decir, la interacción social de tipo colaborativa y dialógica, se considera deseable.

Estas cualidades debieran permitir que el residente adquiera un mayor protagonismo y una participación activa, para lo cual resulta fundamental que el docente reconozca y valide de este modo la experiencia, los conocimientos, las habilidades y el criterio del residente. De esta manera, estas características deseables en el plano humano permiten hacer más efectiva esta estrategia de enseñanza, es decir, se logra de mejor modo el objetivo principal, el de motivar el aprendizaje y autonomía del aprendiz.

La calidad de la relación social resulta ser fundamental para el operar óptimo de la retroalimentación, ya que viene a ser una condicionante que le permite al residente interpretar de modo constructivo cualquier forma de interacción que se dé, incluso al ser señalados aspectos no logrados e insuficientes de su desempeño.

Los hallazgos anteriores vienen a complementar la literatura existente, ya que en ella escasamente se señala la relevancia de la dimensión de la relación social involucrada e inevitable, por lo 
cual se estima que muchos aspectos del proceso efectivo de la retroalimentación se relativizan según la calidad de la interacción social.

Otros estudios y trabajos deberán explicar aún más las habilidades que fomenten el diálogo y la colaboración constructiva en la formación de especialistas, a fin de hacerlas más específicas, mensurables, evaluables, y por ende, entrenables.

Por lo tanto, la retroalimentación en la formación en el área de la Psiquiatría, como en otras áreas de la medicina y en otras áreas de la salud mental, requerirá supervisores y docentes con habilidades interaccionales, dialógicas y colaborativas, las que de estar escasamente desarrolladas, deberán ser entrenadas.

\section{Referencias}

1. Ende J. Feedback in Clinical Medical Education. JAMA 1983; 250 (6): 777-81.

2. Hattie J, Timperley H. The power of feedback. Rev Educ Res 2007; 77 (1): 81-112.

3. Rolfe I, MacPherson J. Formative assessment: how am I doing? Lancet 1995; 345: 837-9.

4. Veloski J, Boex J, Grasberger M, Evans A, Wolfson D. Systematic review of the literature on assessment, feedback and physicians clinical performance: BEME Guide No 7. Med Teach 2006; 28: 117-28.

5. Kianmehr N, Mofidi M, Yazdanpanah R, Ahmadi M. Medical student and patient perspectives on bedside teaching. Saudi Med J 2010; 31 (5): 565-8.
6. Salam A, Halizah H, Mohamad N, Das S, Yousuf R. Bedside teaching in undergraduate medical education: Issues, strategies, and new models for better preparation of new generation doctors. Iran J Med Sci 2011; 36 (1): 1-6.

7. Archer JC. State of the science in health professional education: effective feedback. Med Educ 2010; 44: 101-8.

8. Isaacson J, Posk L, Liaker D, Halperin A. Resident perceptions of the evaluative process. J Gen Intern Med 1995; 10 (suppl): 89.

9. Delva D, Sargeant J, Miller S, Holland J, Brown P, Leblanc C, et al. Encouraging residents to seek feedback. Med Teach 2013; 35 (12): e1625-31.

10. Bose MM, Gijselaers WH. Why supervisors should promote feedback-seeking behavior in medical residency. Med Teach 2013; 35 (11): 1573-83.

11. Bing-You RG, Paterson J, Levine MA. Feedback falling on deaf ears: residents' receptivity to feedback is tempered by sender credibility. Med Teach 1997; 19 (1): 40-4.

12. Glaser B, Strauss A. The discovery of grounded theory. Chicago: Aldine; 1967.

13. Mella O. Metodología cualitativa en ciencias sociales $y$ educación. Santiago de Chile: Editorial Primus; 2003.

14. Strauss A, Corbin J. Basics of qualitative research. Newbury-London-New Delhi: Sage; 1990.

15. Krause M. La reconstrucción de la estructura interna de las representaciones sociales a través de un análisis cualitativo y relacional. En Memorias de la Cuarta conferencia internacional sobre representaciones sociales. Ciudad de México: Universidad Autónoma Metropolitana; 1998.

16. Flick U. Introducción a la investigación cualitativa. Madrid: Ediciones Morata; 2004. 